\title{
PPAR $\alpha$ activation alleviates damage to the cytoskeleton during acute myocardial ischemia/reperfusion in rats
}

\author{
JIE YUAN ${ }^{1,2}$, HONGDAN MO ${ }^{1,2}$, JING LUO $^{1,2}$, SUHONG ZHAO $^{1,2}$, \\ SHUANG LIANG ${ }^{1,2}$, YU JIANG ${ }^{1,2}$ and MAOMAO ZHANG ${ }^{1,2}$ \\ ${ }^{1}$ Department of Cardiology, The Second Affiliated Hospital of Harbin Medical University; \\ ${ }^{2}$ The Key Laboratory of Myocardial Ischemia, Harbin Medical University, \\ Ministry of Education, Harbin, Heilongjiang 150001, P.R. China
}

Received October 2, 2017; Accepted January 16, 2018

DOI: $10.3892 / \mathrm{mmr} .2018 .8771$

\begin{abstract}
The cytoskeleton serves an important role in maintaining cellular morphology and function, and it is a substrate of calpain during myocardial ischemia/reperfusion (I/R) injury (MIRI). Calpain may be activated by endoplasmic reticulum (ER) stress during MIRI. The activation of peroxisome proliferator-activated receptor $\alpha(\operatorname{PPAR} \alpha)$ may inhibit ischemia/reperfusion damage by regulating stress reactions. The present study aimed to determine whether the activation of PPAR $\alpha$ protects against MIRI-induced cytoskeletal degradation, and investigated the underlying mechanism involved. Wistar rats were pretreated with or without fenofibrate and subjected to left anterior descending coronary artery ligation for $45 \mathrm{~min}$, followed by $120 \mathrm{~min}$ of reperfusion. Calpain activity and the expression of PPAR $\alpha$, desmin and ER stress parameters were evaluated. Electrocardiography was performed and cardiac function was evaluated. The ultrastructure was observed under transmission electron microscopy. $\mathrm{I} / \mathrm{R}$ significantly induced damage to the cytoskeleton in cardiomyocytes and cardiac dysfunction, all of which were improved by PPAR $\alpha$ activation. In addition, I/R increased ER
\end{abstract}

Correspondence to: Dr Jie Yuan, Department of Cardiology, The Second Affiliated Hospital of Harbin Medical University, 246 Xuefu Road, Harbin, Heilongjiang 150001, P.R. China

E-mail: 13936336061@163.com

Abbreviations: AMPK, AMP activated protein kinase; ER, endoplasmic reticulum; GRP78, glucose-regulated protein-78; $\mathrm{I} / \mathrm{R}$, ischemia/reperfusion; LAD, left thoracotomy and the left anterior descending; LVDd, left ventricular end-diastolic diameter; LVEF, left ventricular ejection fraction; PPAR $\alpha$, peroxisome proliferator-activated receptor $\alpha$; ROS, reactive oxygen species; RXR, retinoid X receptor; SERCA, sarcoplasmic reticulum $\mathrm{Ca}^{2+}$ ATPase; Suc-LLVY-AMC, Suc-Leu-Leu-ValTyr-7-amino-4-methylcoumarin; MIRI, myocardial ischemia/reperfusion injury; ECG, electrocardiogram

Key words: PPAR $\alpha$, cytoskeleton, desmin, acute MIRI, endoplasmic reticulum stress, calpain stress and calpain activity, which were significantly decreased in fenofibrate-pretreated rat heart tissue. The results suggested that PPAR $\alpha$ activation may exert a protective effect against $\mathrm{I} / \mathrm{R}$ in the myocardium, at least in part via ER stress inhibition. Suppression of ER stress may be an effective therapeutic target for protecting the I/R myocardium.

\section{Introduction}

The cytoskeleton is a reticular structure involved in maintaining cellular morphology and physiological function, and it is susceptible to the calpain system, which may be activated by high concentrations of calcium during myocardial ischemia/reperfusion (I/R) injury (MIRI). The endoplasmic reticulum (ER) is a dynamic membranous network which is involved in various physiological processes, including protein synthesis, steroid hormone synthesis, intracellular calcium homeostasis regulation and molecular signal transmission (1). ER stress that disrupts ER function may occur in response to radiation, ischemic stress, glucose and lipid metabolic disorders, and other cellular stressors, which lead to the accumulation of unfolded and misfolded proteins in the ER and calcium dyshomeostasis. In the early stages of ER stress, the ER may decrease the damage to cells caused by unfolded and misfolded proteins, via activation of transcriptional and translational pathways. However, sustained or serious ER stress may cause alterations in the lipid composition and calcium reserves of the ER, and cytoskeletal degradation and inflammation may be induced by the imbalance of homeostasis in the cells (2). Studies have confirmed that ER stress is implicated in the pathogenesis of diseases, including I/R injury, shock and cancer $(3,4)$. Previously, reperfusion injury was significantly alleviated by ER stress inhibitors in an in vivo model of liver, myocardial and cerebral acute I/R (5-7).

Peroxisome proliferator-activated receptor $\alpha(\operatorname{PPAR} \alpha)$ is a subtype of transcription factors of the nuclear receptor superfamily. It is highly expressed in various metabolically active organs, including the liver, heart and kidney. PPAR $\alpha$ serves a critical biological role in regulating the expression of target genes by forming heterodimers with the retinoid $\mathrm{X}$ receptor, including genes involved in mitochondrial $\beta$-oxidation, ketogenesis, lipoprotein transport and glycolysis (8). A previous 
study revealed that, besides regulating energy metabolism and inflammatory responses, $\operatorname{PPAR} \alpha$ serves a key role in intervening in the occurrence and development of disease by regulating stress reactions (9). Research in the liver has shown that ER stress may be suppressed by activating PPAR $\alpha$; in fatty liver tissue, PPAR $\alpha$ activation reduced the accumulation of lipid droplets and apolipoprotein B-100 in the hepatic ER, corrected the disturbance in the lipid composition of the ER and upregulated the expression of sarcoplasmic reticulum $\mathrm{Ca}^{2+}$ ATPase, resulting in the prevention of the induction of ER stress and an improvement in hepatic steatosis associated with fructose consumption (10). In addition, PPAR $\alpha$ was associated with the prevention of ER stress induced by the disturbance of lipid metabolism in cardiac cells by enhancing AMP-activated protein kinase activity (11). A previous report demonstrated that PPAR $\alpha$ activation may alleviate acute I/R-induced injury to the mitochondrial ultrastructure, and the underlying protective mechanism involves the antioxidant effect of PPAR $\alpha$ (12). However, there are few studies that demonstrate the protective effect of the PPAR $\alpha$ on the cytoskeleton and ER stress during MIRI. Therefore, the present study aimed to determine the protective effect of PPAR $\alpha$ activation on myocardial I/R injury in rats and to investigate its possible underlying mechanisms in ER stress. The present study demonstrated the protective effects of fenofibrate on I/R heart tissue through ER stress inhibition in the myocardium. These results provide a theoretical basis for future clinical trials for fenofibrate in patients with reperfusion injury.

\section{Materials and methods}

Animals. A total of 48 male Wistar rats (age, 6-8 weeks; weight, 160-220 g) were bred in-house (Laboratory Animal Center, The Second Affiliated Hospital of Harbin Medical University, Harbin, China) and had ad libitum access to food and water. Rats were maintained at a controlled temperature $\left(22 \pm 2^{\circ} \mathrm{C}\right)$ and humidity $(50-70 \%)$. All animals were housed in a controlled pathogen free environment with 12-h light and dark cycles. All protocols for animals were performed strictly in accordance with the guidelines for the Care and Use of Laboratory Animals, and the present study was approved by the Ethical Committee of The Second Affiliated Hospital of Harbin Medical University.

Myocardial I/R injury model. Wistar rats were randomly divided into four groups ( $\mathrm{n}=8 \mathrm{rats} /$ group): Normal group (normal); sham group (sham); I/R group (I/R); and fenofibrate pretreatment $+\mathrm{I} / \mathrm{R}$ group $(\mathrm{FF}+\mathrm{I} / \mathrm{R})$. Fenofibrate (Sigma-Aldrich; Merck KGaA, Darmstadt, Germany) was suspended in 3\% gum acacia (Sigma-Aldrich; Merck KGaA) and administered for 7 days at a dose of $80 \mathrm{mg} \cdot \mathrm{kg}^{-1} \cdot \mathrm{day}^{-1}$ by oral gavage in the $\mathrm{FF}+\mathrm{I} / \mathrm{R}$ group. The other three groups of rats were given a similar amount of the solvent (3\% gum acacia) for 7 days. The dose of fenofibrate and the concentration of gum acacia were based on a previous study $(12,13)$.

A total of $1 \mathrm{~h}$ subsequent to the final intragastric administration, the myocardial I/R model was generated. Briefly, rats were intraperitoneally injected with $45 \mathrm{mg} / \mathrm{kg}$ pentobarbital sodium. Following oral endotracheal intubation, the rats were mechanically ventilated with air using a rodent ventilator (room air; rate, 75 cycles $/ \mathrm{min} ; 3 \mathrm{ml} / 100 \mathrm{~g}$ tidal volume). The electrocardiogram (ECG) was recorded. The left anterior descending (LAD) coronary artery was ligated (1-2 mm region under the boundary pulmonary artery pyramid and left auricle of heart) with a 5-0 polyester suture. A small polyethylene tube was placed between the ligature and the myocardial tissues. The rats in the sham group underwent the same surgical procedures, although the suture was not fastened. Following ischemia for $45 \mathrm{~min}$, the ligature was released to permit reperfusion for $120 \mathrm{~min}$. At the end of reperfusion, the rats were sacrificed by overdose of pentobarbital sodium $(100 \mathrm{mg} / \mathrm{kg})$, and the left ventricle tissues were harvested and frozen in liquid nitrogen $\left(-196^{\circ} \mathrm{C}\right)$ immediately for further measurement.

Heart functional examination. Standard echocardiography was performed at room temperature for all groups of rats following 45 min acute myocardial ischemia and $120 \mathrm{~min}$ reperfusion. The left ventricular ejection fraction (LVEF) and left ventricular end-diastolic diameter (LVDd) were measured from the parasternal long-axis view at the mid-papillary muscle level with an ultrasound imaging system (Vevo770; FUJIFILM Visual Sonics, Inc., Toronto, ON, Canada).

Electron microscopy. Fresh myocardial tissues $\left(1 \mathrm{~mm}^{3}\right)$ were excised from the tissue of the cardiac apex following reperfusion, and fixed overnight in $3 \%$ glutaric dialdehyde at $4{ }^{\circ} \mathrm{C}$. Trimmed tissues were post-fixed with $1 \%$ osmium tetraoxide for $2 \mathrm{~h}$. Samples were subsequently dehydrated in ethanol followed by acetone and embedded in Epoxy resin (Ladd Research Industries, Williston, VT, USA). The specimens were processed into ultrathin sections $(50 \mathrm{~nm})$. The sections were stained with $1 \%$ uranium acetate and $1 \%$ lead citrate for 10 min respectively at room temperature. A total of 64 tissue sections (eight fields of view per section), corresponding to four sections from each rat $(n=4)$, were observed via a transmission electron microscope (H-7650; Hitachi, Ltd., Tokyo, Japan) at an accelerating voltage of $80 \mathrm{kV}$.

Reverse transcription-quantitative polymerase chain reaction (RT-qPCR). Total RNA was extracted using TRIzol reagent (Life Technologies; Thermo Fisher Scientific, Inc., Waltham, MA, USA), and the RNA concentration was determined using a UV spectrophotometer. RT of total RNA to cDNA was performed using an AccuPower RocketScript ${ }^{\text {TM }}$ RT PreMix kit (Bioneer Corporation, Daejeon, Korea), following the manufacturer's protocol. The thermal cycle profile for RT was set for primer annealing at $37^{\circ} \mathrm{C}$ for $10 \mathrm{~min}$, cDNA synthesis at $50^{\circ} \mathrm{C}$ for $60 \mathrm{~min}$ and heat inactivation at $95^{\circ} \mathrm{C}$ for $5 \mathrm{~min}$. qPCR was performed using an AccuPower 2x GreenStar ${ }^{\mathrm{TM}}$ qPCR Master Mix kit (Bioneer Corporation) in a Bio-Rad iQ5 optical module (Bio-Rad Laboratories, Inc., Hercules, CA, USA). qPCR was performed under the following conditions: $95^{\circ} \mathrm{C}$ for $10 \mathrm{~min}$, and 40 cycles of denaturation at $95^{\circ} \mathrm{C}$ for $10 \mathrm{sec}$ and annealing at $58^{\circ} \mathrm{C}$ for $20 \mathrm{sec}$. Primer sequences were: GRP78 forward, 5'-TGACTATGAAGAATCCCAAGA-3' and reverse, 5'-TATCAACATCCAGTTCCACC-3'); GAPDH forward, 5'-GTTCAACGGCACAGTCAAGG-3' and reverse, 5'-CAC CAGTGGATGCAGGGAT-3'. $2^{-\Delta \Delta C q}$ was calculated for every sample, and the mRNA expression levels were determined using the $2^{-\triangle \Delta \mathrm{Cq}}$ method (14) and normalized to GAPDH. 

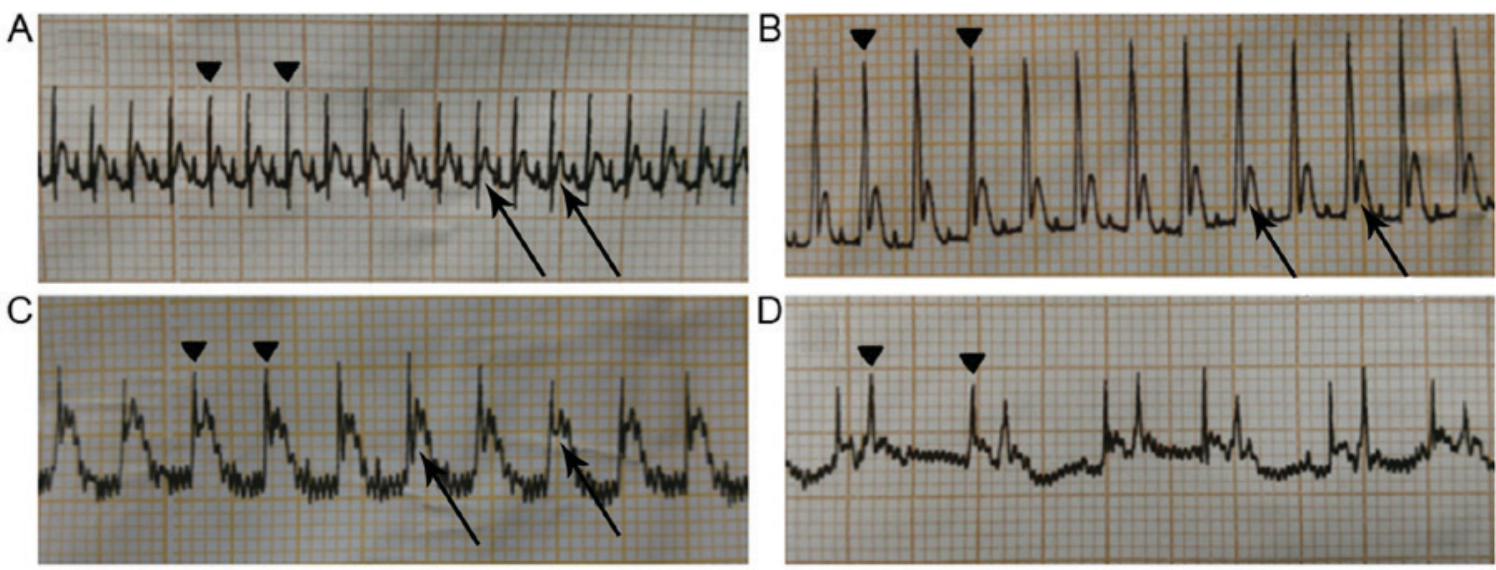

Figure 1. Representative ECG results. (A) A normal ECG was observed in the normal and sham group. (B) QRS wave amplitude increased, (C) the ST segment was elevated and (D) reperfusion arrhythmia occurred following arterial ligation in the I/R and fenofibrate+I/R groups. The black triangle indicates the QRS wave and the black arrow indicates the ST segment. ECG, electrocardiogram; I/R, ischemia/reperfusion.

Western blotting. Heart tissues were homogenized in radioimmunoprecipitation assay lysis buffer (Beyotime Institute of Biotechnology, Haimen, China). The homogenates were centrifuged at $1,600 \mathrm{x}$ g for $10 \mathrm{~min}$ at $4{ }^{\circ} \mathrm{C}$. A bicinchoninic acid assay kit (Beyotime Institute of Biotechnology) was used for protein quantification. A total of $20 \mu \mathrm{g}$ protein was electrophoresed on 10-15\% SDS-PAGE gels and transferred onto polyvinylidene fluoride membranes (EMD Millipore, Billerica, MA, USA). Membranes were subsequently blocked with $5 \%$ (w/v) non-fat milk in TBS containing $0.1 \%(\mathrm{v} / \mathrm{v})$ Tween-20 and incubated overnight at $4^{\circ} \mathrm{C}$ with anti-PPAR $\alpha$ (cat no. WL00978; 1:1,000; Wanleibio, Co., Ltd., Shanghai, China), anti-Desmin (cat no. ab32362; 1:8,000; Abcam, Cambridge, UK), anti-GRP78 (cat no. WL00621; 1:1,000; Wanleibio, Co., Ltd.), and anti-GADPH (cat no. ab181602; 1:8,000; Abcam). Following washing, bound antibodies were detected following incubation for $1 \mathrm{~h}$ at room temperature with peroxidase-conjugated goat anti-rabbit IgG (cat no. ZB-2301; 1:10,000; OriGene Technologies, Inc., Beijing, China). Blots were developed using Western Lightning BeyoECL Plus reagent (Beyotime Institute of Biotechnology) and were quantified using ImageJ software (version 2.1.4.7; National Institutes of Health, Bethesda, MD, USA).

Measurement of calpain activity. Calpain activity was measured using the GENMED Tissue Calpain Activity Assay kit (GenMed Scientifics, Inc., Wilmington, DE, USA). A total of $100 \mathrm{mg}$ tissue was homogenized in $1,000 \mu 1$ nondenaturing lysis buffer provided with the kit, the lysates were centrifuged for $15 \mathrm{~min}$ at $12,000 \mathrm{x} \mathrm{g}$ and the supernatant was collected. Protein concentrations were quantified using a micro bicinchoninic acid protein assay. Suc-Leu-Leu-Val-Tyr-7-amino-4-methylcoumarin (Suc-LLVY-AMC) was used as the calpain substrate. A total of $50 \mu \mathrm{l}$ supernatant $(100 \mu \mathrm{g}$ protein $/ 50 \mu \mathrm{l})$ was added to $150 \mu \mathrm{l}$ GENMED substrate (dissolved in GENMED buffer), and $\mathrm{AMC}$ release was measured using a microplate reader (Thermo Fisher Scientific, Inc.) using $380 \mathrm{~nm}$ excitation and $430 \mathrm{~nm}$ emission filters. Calpain activity was expressed as $\mu \mathrm{mol} \mathrm{AMC} \mathrm{released} / \mathrm{mg}$ tissue protein at $37^{\circ} \mathrm{C}$ and in $\mathrm{pH} 7.5$ per hour. The calcium activity levels in the different experimental groups were comparable.
Statistical analysis. All data are expressed as the mean \pm standard error. All the statistical analyses were performed using SPSS version 20.0 software (IBM Corp., Armonk, NY, USA). All P-values were two sided, and $\mathrm{P}<0.05$ was considered to indicate a statistically significant difference. Multiple group comparisons were analyzed using one-way analysis of variance, and the post hoc test employed was Fisher's least significant difference test. The Pearson correlation method was performed to analyze the association between the expression of GRP78 protein and desmin protein.

\section{Results}

ECG of rats with acute I/R. Prior to LAD ligation, the ECG of each rat in the I/R and $\mathrm{FF}+\mathrm{I} / \mathrm{R}$ groups was normal, as those in normal and sham group (Fig. 1A). Following LAD ligation, the distal myocardium may turn white or cyanosed. The ventricular wall motion decreased and the ECG demonstrated that the amplitude of the QRS wave increased significantly (Fig. 1B) and the ST segment was elevated (Fig. 1C). Following reperfusion, the myocardium was hyperemic and the cyanotic color disappeared. The ST segment resolution and amplitude of QRS decreased, which were additionally observed in the ECG. There are certain studies that suggest that the ECG (such as 'Wagner QRS score') may be used to estimate myocardial infarct size. However, arrhythmias, including left/right bundle branch block and ventricular paced ECG, are the confounders of QRS score calculation (15-17). In the present study, certain rats of I/R group and $\mathrm{FF}+\mathrm{I} / \mathrm{R}$ group exhibited arrhythmia waves in the ECG following reperfusion during the experiments (Fig. 1D); the most common reperfusion arrhythmias were ventricular tachycardia, atrioventricular block and sinoatrial block. The present study concluded that reperfusion arrhythmia may disturb the QRS score calculation, and therefore did not consider using the ECG to evaluate the degree of myocardial infarction, and it was only one of the indicators to determine whether or not the model was established successfully.

Cardiac function of each group. The cardiac function of each rat $(n=8)$ was examined by echocardiography. Compared with the normal group, the values of LVEF and LVDd had no 
A

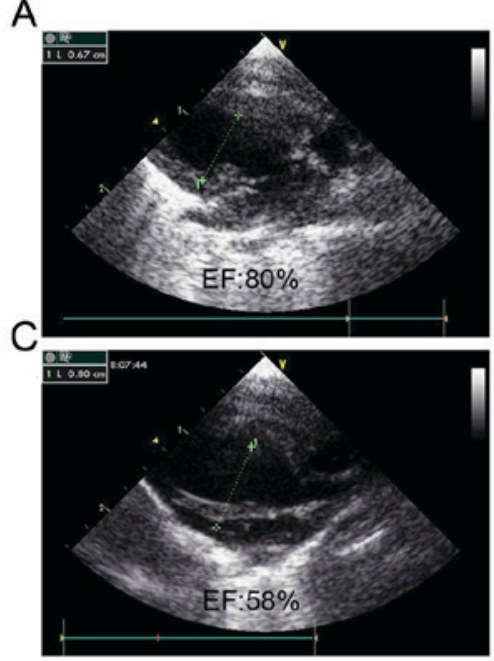

B

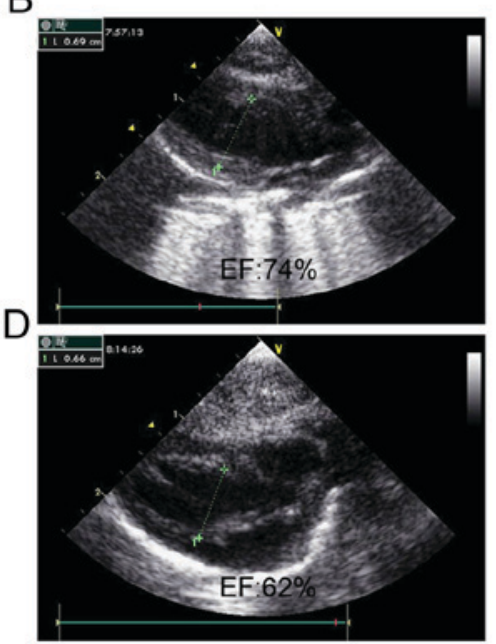

E

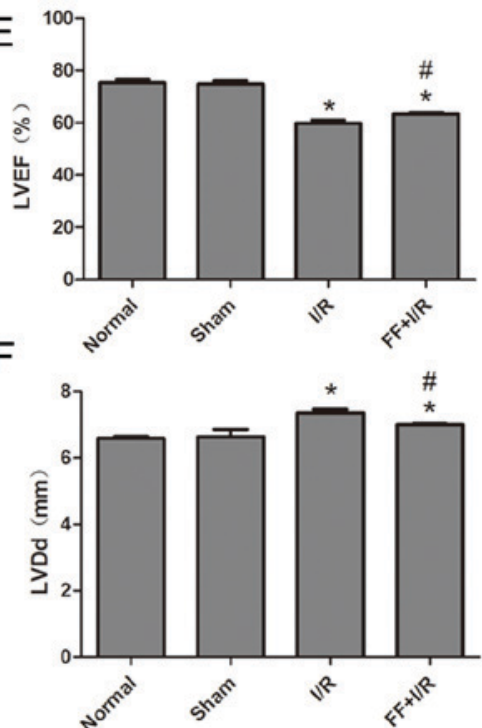

Figure 2. Effect of FF on cardiac function in rats with acute I/R. Echocardiograms of rats in the (A) normal group, (B) Sham group, (C) I/R group and (D) $\mathrm{FF}+\mathrm{I} / \mathrm{R}$ group. (E) The LVEF of rats in each group. (F) The LVDd of rats in each group. Data are presented as the mean \pm standard error ( $=8$ ). ${ }^{*} \mathrm{P}<0.05$ vs. sham group; ${ }^{\#} \mathrm{P}<0.05$ vs. I/R group. I/R, ischemia/reperfusion; FF, pretreatment with fenofibrate; EF, ejection fraction; LVDd, left ventricular end-diastolic diameter; LVEF, left ventricular ejection fraction.

significant differences in the sham group (normal, $75.25 \pm 3.81$ vs. sham, $74.75 \pm 3.61, \mathrm{P}>0.05$; normal, $6.59 \pm 0.16$ vs. sham, $6.64 \pm 0.60, \mathrm{P}>0.05)$. Compared with the sham group, the values of $\mathrm{LVEF}$ in the I/R group and $\mathrm{FF}+\mathrm{I} / \mathrm{R}$ group were lower, while the LVDd values were higher (all $\mathrm{P}<0.05$ ). Furthermore, compared with the I/R group, the values of LVEF in the FF+I/R group were higher (I/R, 59.75 \pm 3.62 vs. FF+I/R, 63.25 \pm 2.05 , $\mathrm{P}<0.05)$, and the LVDd values were lower $(\mathrm{I} / \mathrm{R}, 7.33 \pm 0.16$ vs. FF+I/R, 7.00 $\pm 0.19, \mathrm{P}<0.05$ ) (Fig. 2).

PPAR $\alpha$ activation decreases cytoskeletal structure damage caused by acute myocardial I/R injury. Fresh myocardial tissues $\left(1 \mathrm{~mm}^{3}\right)$ were excised from the cardiac apex following reperfusion, and the mitochondrial ultrastructure was observed by transmission electron microscopy $(n=4)$. Regularly arranged endoplasmic reticulum, mitochondria and complete $\mathrm{Z}$ lines were observed in the normal and sham groups (Fig. 3A and B). In the I/R group, the transmission electron microscopy observation revealed destruction of the cytoskeleton and organelles, including partial and even total rupture, in addition to disintegration of the $\mathrm{Z}$ lines, dilation of the endoplasmic reticulum and turgidity of the mitochondria, accompanied by distinct dislocation of the endoplasmic reticulum and mitochondria (Fig. 3C). Notably, pretreatment with fenofibrate alleviated these deleterious effects on the cardiac subcellular structure induced by I/R injury (Fig. 3D).

PPAR $\alpha$ activation decreases the degradation of desmin caused by acute myocardial I/R injury. The expression of desmin protein was detected by western blotting $(n=8)$. Compared with the normal group, the expression of desmin protein in the myocardium was not significantly different in the sham group (normal, $0.43 \pm 0.02$ vs. sham, $0.42 \pm 0.03 ; \mathrm{P}>0.05)$. Compared with the sham group, I/R induced a decrease in the expression levels of desmin, a sensitive substrate of calpain (18), while such alterations were suppressed by pretreatment with fenofibrate (I/R, $0.25 \pm 0.04$ vs. FF+I/R, 0.30 $\pm 0.03 ; \mathrm{P}<0.01 ;$ Fig. 4).

PPAR $\alpha$ levels in each group. The expression of PPAR $\alpha$ was measured by western blotting $(n=8)$. Compared with the normal group, the expression of PPAR $\alpha$ protein in the myocardium was not significantly different compared with the sham group (normal, $0.48 \pm 0.04$ vs. sham, 0.47 \pm 0.03 ; P>0.05). Compared with the sham group, I/R caused marked decreases in PPAR $\alpha$ protein expression levels $(\mathrm{P}<0.001)$, but it was significantly

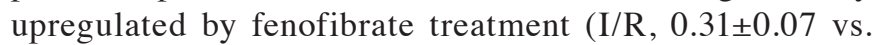
I/R+FF, 0.44士0.05; P<0.001; Fig. 5).

Calpain activity in myocardium. Calpain activity was measured in each group $(n=8)$. Compared with the normal group, there was no significant difference in calpain activity in the sham group (normal, 5.21 \pm 0.61 vs. sham, 5.65 \pm 0.67 ; P>0.05). I/R injury had resulted in a marked increase in calpain activity compared with that in non-I/R hearts $(\mathrm{P}<0.001)$, whereas the increased calpain activity was significantly attenuated by pretreatment with fenofibrate $(\mathrm{I} / \mathrm{R}, 11.47 \pm 1.19 \mathrm{vs} . \mathrm{FF}+\mathrm{I} / \mathrm{R}$, 8.49 \pm 1.44 ; $\mathrm{P}<0.001$; Fig. 6).

PPAR $\alpha$ activation inhibits GRP78 overexpression caused by acute myocardial I/R injury. I/R injury in the tissues has been demonstrated to be associated with ER stress induction. Thus, possible alterations in the mRNA and protein expression of ER stress parameters were evaluated $(n=8)$. As presented in Fig. 7, compared with the normal group, there was no difference in the expression of GRP78 mRNA and protein in the sham group (normal, $0.98 \pm 0.06$ vs. sham, $1.00 \pm 0.07, \mathrm{P}>0.05$; normal, $0.30 \pm 0.04$ vs. sham, $0.30 \pm 0.04$, $\mathrm{P}>0.05)$. Compared with the sham group, the expression of GRP78 mRNA and protein was elevated by I/R $(\mathrm{P}<0.001)$, and expression was reduced by pretreatment with the PPAR $\alpha$ 

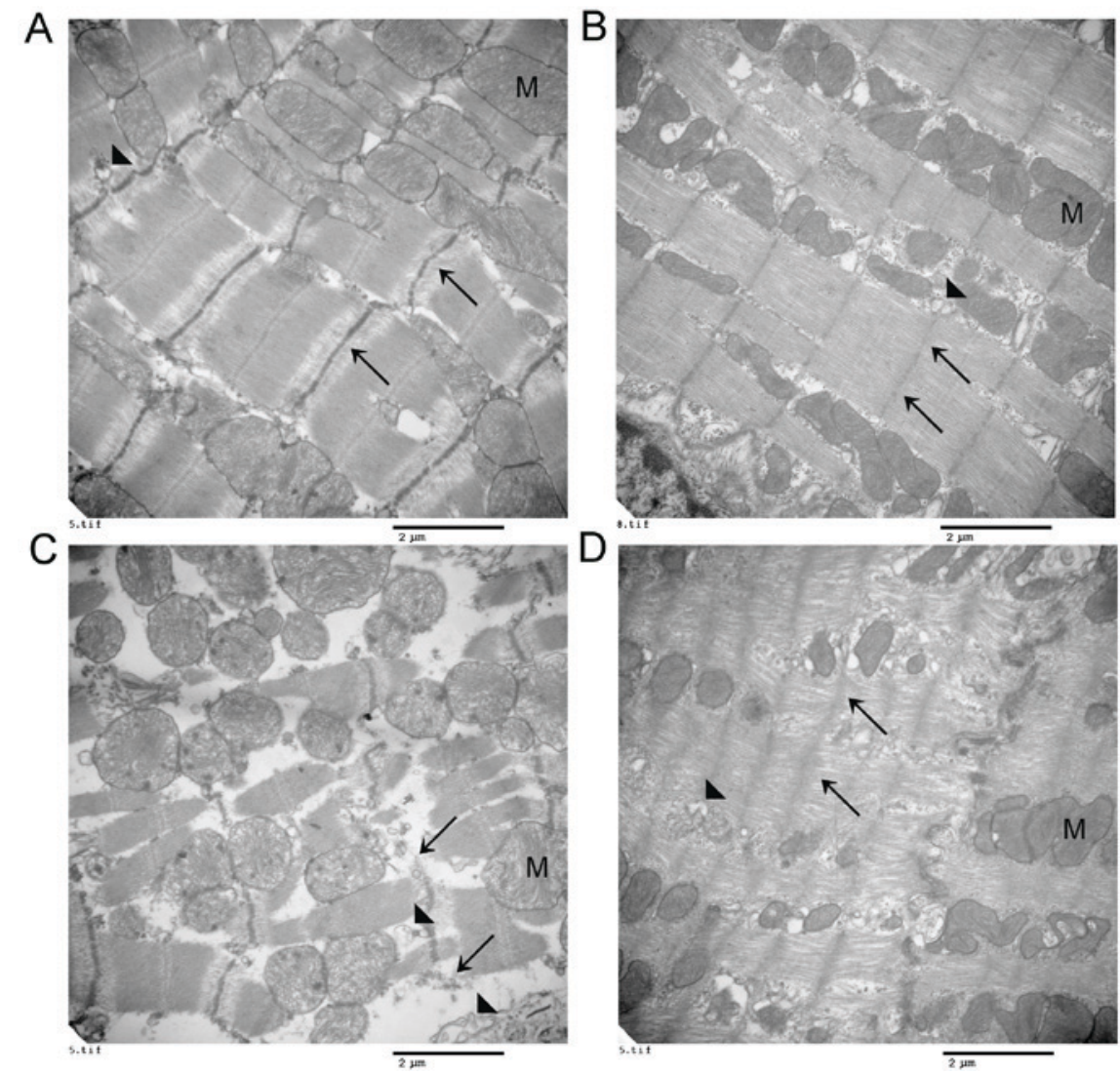

Figure 3. Influence of FF on the ultrastructure of myocardial tissues of rats with acute $\mathrm{I} / \mathrm{R}$. Representative electron micrographs of cardiomyocytes of rats in each group ( $\mathrm{n}=4 \mathrm{rats} / \mathrm{group}$ ). The black triangle indicates the endoplasmic reticulum, the arrow indicates the $\mathrm{Z}$ line and the $\mathrm{M}$ indicates mitochondria. (A) Normal group. (B) Sham group. (C) I/R group. (D) FF+I/R group. Scale bar=2 $\mu \mathrm{m}$. I/R, ischemia/reperfusion; FF, pretreatment with fenofibrate.
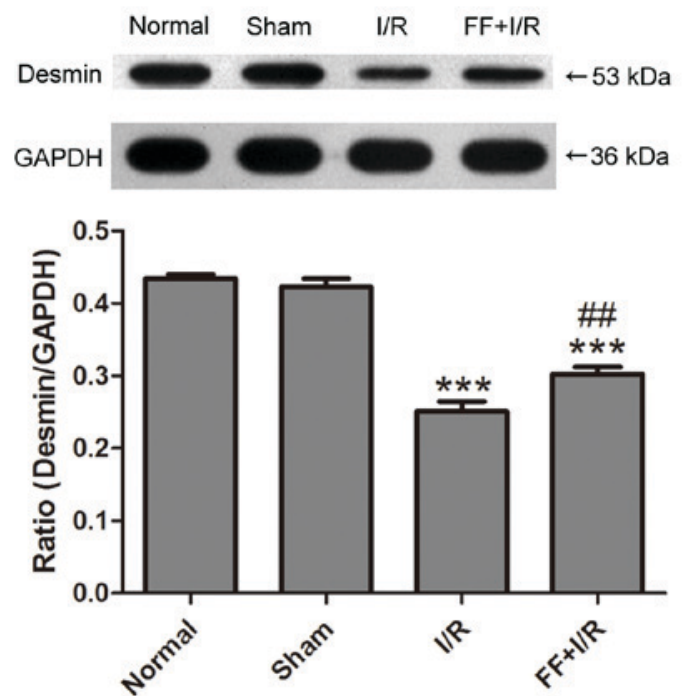

Figure 4. PPAR $\alpha$ activation decreases degradation of desmin caused by acute myocardial I/R injury. Expression levels of the desmin protein were detected by western blotting. A representative image is presented. Data are presented as the mean \pm standard error $(n=8) .{ }^{* * * *} \mathrm{P}<0.001$ vs. sham group; ${ }^{\# \#} \mathrm{P}<0.01$ vs. $\mathrm{I} / \mathrm{R}$ group. I/R, ischemia/reperfusion; FF, pretreatment with fenofibrate; PPAR $\alpha$, peroxisome proliferator-activated receptor $\alpha$.

agonist fenofibrate (I/R, $1.76 \pm 0.11$ vs. FF+I/R, $1.33 \pm 0.16$, $\mathrm{P}<0.001 ; \mathrm{I} / \mathrm{R}, 0.59 \pm 0.07$ vs. $\mathrm{FF}+\mathrm{I} / \mathrm{R}, 0.43 \pm 0.07, \mathrm{P}<0.001$; Fig. 7).
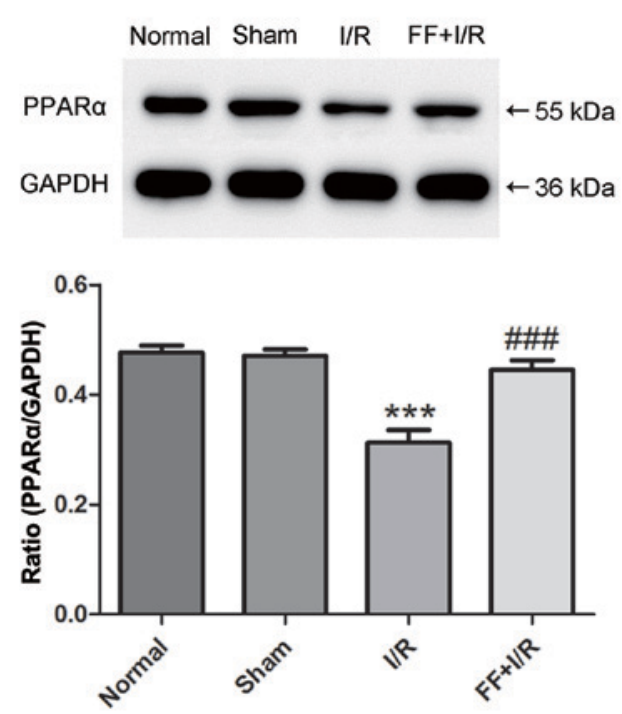

Figure 5. Effect of FF on the expression of PPAR $\alpha$ protein in the myocardial tissue of rats with acute I/R. The expression levels of the PPAR $\alpha$ protein were detected by western blotting. A representative image is presented. Data are presented as the mean \pm standard error $(n=8) .{ }^{* * * *} \mathrm{P}<0.001$ vs. sham group.

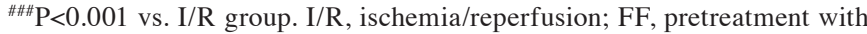
fenofibrate; PPAR $\alpha$, peroxisome proliferator-activated receptor $\alpha$.

Correlation analysis between the expression of GRP78 protein and desmin protein. Negative correlations were observed between the expression of the GRP78 protein and desmin 


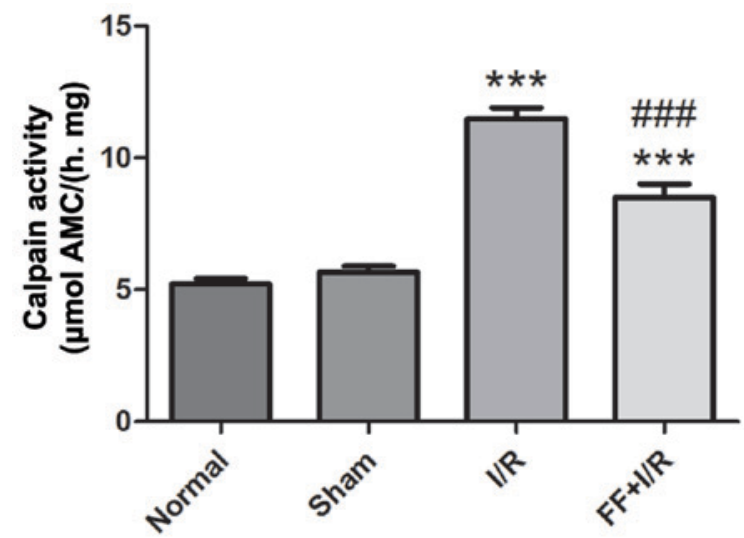

Figure 6. Effect of FF on calpain activity in the myocardium of rats with acute I/R. ${ }^{* * *} \mathrm{P}<0.001$ vs. sham group. ${ }^{\# \#} \mathrm{P}<0.001$ vs. I/R group. I/R ischemia/reperfusion; FF, pretreatment with fenofibrate.

protein in the $\mathrm{I} / \mathrm{R}$ group and $\mathrm{FF}+\mathrm{I} / \mathrm{R}$ group $(\mathrm{I} / \mathrm{R}, \mathrm{r}=-0.725$, $\mathrm{P}<0.05 ; \mathrm{FF}+\mathrm{I} / \mathrm{R}, \mathrm{r}=-0.769, \mathrm{P}<0.05 ;$ Fig. 8).

\section{Discussion}

In order to evaluate the severity of cardiac damage, the present study observed the myocardial cytoskeleton structure of a myocardial I/R injury rat model which was generated by a LAD coronary artery ligation for $45 \mathrm{~min}$ and reperfusion for $120 \mathrm{~min}$. Following LAD ligation, the distal myocardium turned white or cyanosed, ventricular wall motion decreased, and the ECG illustrated that the amplitude of the QRS wave increased significantly and the ST segment was elevated. Following reperfusion, the myocardium was hyperemic and the cyanotic color disappeared. ST segment resolution, a decrease in the amplitude of the QRS wave and reperfusion arrhythmia were additionally observed on the ECG, which confirmed that the acute MIRI models were successfully established. Myocardial injury in the I/R group was more serious than that in the normal and sham groups; swelling and absence of the $\mathrm{Z}$ line were observed ultrastructurally, and the cardiac function was decreased markedly. In addition, it was demonstrated that myocardial PPAR $\alpha$ mRNA and protein expression levels decreased significantly in the I/R group, whereas pretreatment with fenofibrate upregulated the expression of the PPAR $\alpha$ protein, and the subcellular structural injury and cardiac dysfunction were relieved. These experiments suggested that PPAR $\alpha$ activation protected against acute myocardial I/R injury.

Acute myocardial I/R injury is one of the foci of basic research and clinical studies, and its pathogenesis remains to be clarified. Intracellular overproduction of oxygen free radicals and calcium overload have been demonstrated to be important pathological factors of myocardial I/R injury (19). In addition, calcium overload may activate a number of calcium-dependent enzyme systems, including phospholipase and calpain, promote the hydrolysis of membrane phospholipids, which leads to damage to and degradation of the cytoskeleton, and induce cellular morphological damage, dysfunction and remodeling $(20,21)$. In this process, if the degradation of the cytoskeleton may be reduced, the damage to and dysfunction of cells due to reperfusion may be relieved effectively.
Calpain is a neutral cysteine amino acid proteolytic enzyme whose activation depends on the calcium concentration. Calpain exists in various cells with inactive zymogen in normal circumstances, and in pathological conditions, including I/R, the sustained increase in intracellular $\mathrm{Ca}^{2+}$ concentration may lead to the hydrolysis and activation of calpain, resulting in the excessive degradation of its substrates, including desmin and fibronectin (22-24). The present study revealed that myocardial calpain activity was enhanced in the I/R group, and the expression of desmin, a cytoskeletal protein, was significantly decreased compared with the sham group; this suggested severe biodegradation of the cytoskeleton. Desmin is one of the important cytoskeletal structures of the myocardium, and structural damage to desmin, which primarily exists in the $\mathrm{Z}$ line and the intercalated disk of myocardial fibers, severely affects the systolic and diastolic function of muscle fibers (25). In addition, the degradation products of desmin may induce alterations in gene regulation in the nucleus, and therefore cause myocardial remodeling $(21,26)$. In conclusion, the damage to desmin induced by the overactivation of calpain has been identified to be one of the most important factors of myocardial I/R injury. In the present study, it was observed that compared with the I/R group, PPAR $\alpha$ activation effectively reduced the activity of calpain in I/R rat hearts, and the expression levels of desmin were similar to those in the normal and sham groups; this suggested that $\operatorname{PPAR} \alpha$ activation may significantly alleviate calcium overload induced by $I / R$, and protect the myocardial ultrastructure from the 'secondary attack' of alterations in the intracellular environment of the myocardium.

In the process of MIRI, ER stress induced by hypoxia-ischemia of tissues, oxidative stress, acidosis and energy deficiency may continue by disrupting intracellular calcium homeostasis, inducing injury to the I/R myocardium (27). Previous studies have suggested that ER stress and calcium overload are essential prerequisites, and form a vicious circle $(28,29)$. The ER becomes weak due to the swelling of myocardial cells and endothelial cells at the early stage of reperfusion, and ER stress may be aggravated. The calcium reserves of the ER tend to collapse and calcium may be released sustainably. Finally, damage to cells following reperfusion is aggravated by calpain system activation (30). Thus, it may be seen that the overload of calcium is associated with the function of the ER in the myocardium in MIRI rats. The mitigation of ER stress maintains the intracellular calcium homeostasis and reduces cytoskeletal damage. GRP78, as a co-chaperone of ER stress, may bind unfolded proteins which accumulate in the ER and protect cells against ER dysfunction during the early stages of ER stress. Elevated protein expression of GRP78 was positively associated with the intensity of ER stress (31). However, overexpression of GRP78 may not be enough to completely prevent ER stress induced by I/R and, subsequently, structural damage and functional disorders may emerge in the myocardium (32). The present study revealed that GRP78 mRNA and protein expression was increased significantly in the I/R group compared with the sham group, which suggested that I/R induced ER stress in cardiomyocytes, and GRP78 was not sufficient to reduce the myocardial damage caused by ER stress; this phenomenon was consistent with that reported in the literature (32). Studies have demonstrated that 
A

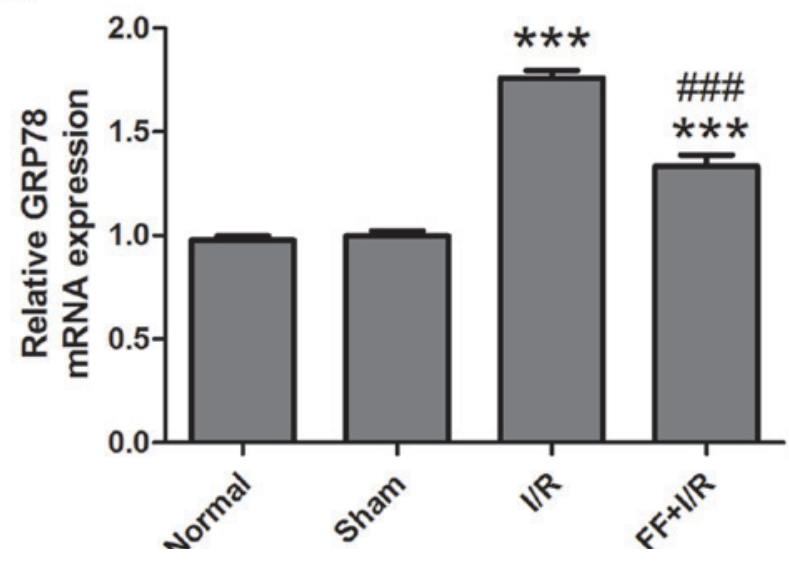

B
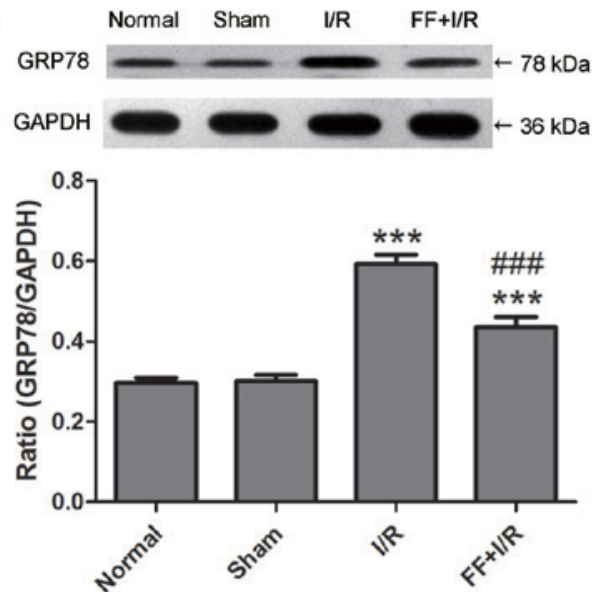

Figure 7. PPAR $\alpha$ activation relieves GRP78 overexpression in the myocardium of rats with acute I/R. Expression levels of GRP78 (A) mRNA and (B) protein were detected by reverse transcription-quantitative polymerase chain reaction and western blotting, respectively. Data are presented as the mean \pm standard error $(\mathrm{n}=8){ }^{. * * *} \mathrm{P}<0.001$ vs. sham group. ${ }^{\# \# \# *} \mathrm{P}<0.01 \mathrm{vs.} \mathrm{I} / \mathrm{R}$ group. PPAR $\alpha$, peroxisome proliferator-activated receptor $\alpha ; \mathrm{I} / \mathrm{R}$, ischemia/reperfusion; FF, pretreatment with fenofibrate; GRP78, glucose-regulated protein-78.
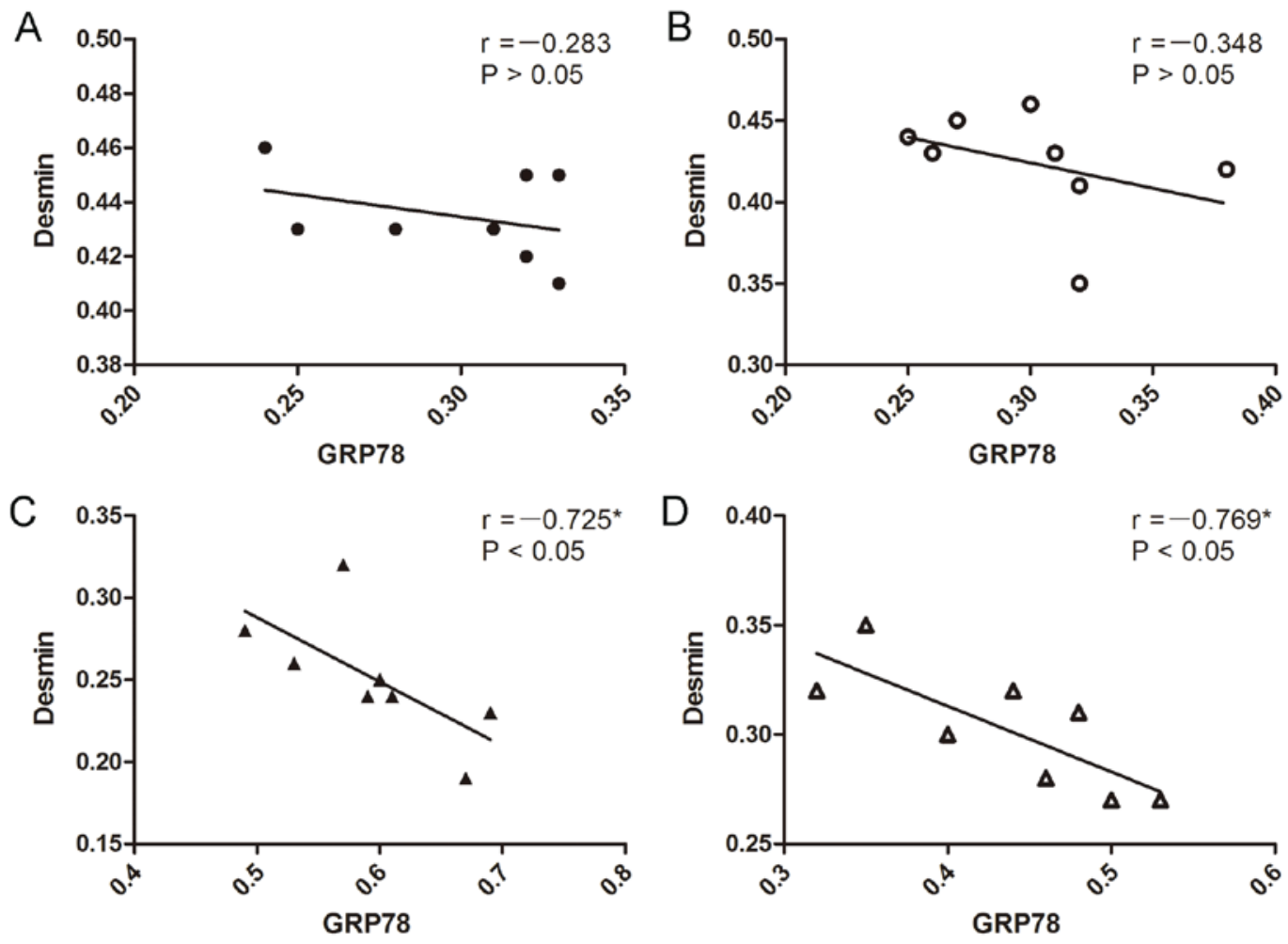

Figure 8. Association between the expression levels of GRP78 and desmin in the myocardium of each group (n=8). (A) Normal group; (B) sham group; (C) I/R group; (D) FF+I/R group. I/R, ischemia/reperfusion; FF, pretreatment with fenofibrate; GRP78, glucose-regulated protein-78.

PPAR $\alpha$ activation may relieve ER stress induced by $I / R$ in the liver and hypertrophied neonatal hearts $(33,34)$. In the present study, the expression of GRP78 mRNA and protein was significantly decreased in the $\mathrm{FF}+\mathrm{I} / \mathrm{R}$ group, which suggested that ER stress was reduced in $\mathrm{FF}+\mathrm{I} / \mathrm{R}$ cardiomyocytes. The trend between the alterations in myocardial calpain activation and the intensity of ER stress was similar in all groups, and a correlation was identified between GRP78 and desmin. Thus, calpain activity alleviation caused by PPAR $\alpha$ activation may be associated with alleviating the intensity of ER stress. The specific underlying mechanism underlying the effect of PPAR $\alpha$ activation on alleviating ER stress requires further research.

Morphological alterations of mitochondria were additionally observed under transmission electron microscopy in the I/R group, including cytoskeletal damage, and PPAR $\alpha$ activation promoted the recovery of the morphology of the mitochondria. These findings were consistent with a previous study (12). A previous study demonstrated that ER stress may induce mitochondrial dysfunction (35). The present study predicts that the effect of PPAR $\alpha$ activation by fenofibrate 
protects against acute myocardial I/R injury, and may be associated with the inhibition of ER stress and ER stress-induced mitochondrial dysfunction in MIRI, although this requires further clarification.

In conclusion, the present study may aid the identification of the underlying mechanisms involved in the protective effects of PPAR $\alpha$ activation on acute myocardial I/R injury. PPAR $\alpha$ activation may suppress I/R-induced ER stress (with a reduction in GRP78 expression), and thus the overactivation of calpain may be prevented, as demonstrated by a reduction in the damage to the cytoskeleton of cardiomyocytes and cardiac dysfunction. The signal transduction involved requires further investigation. Suppression of ER stress may be a new useful target for protecting the I/R myocardium.

\section{Acknowledgements}

Not applicable.

\section{Funding}

The present study was supported by the Open Foundation of Key Laboratory of Myocardial Ischemia, Harbin Medical University, Ministry of Education (China) (grant nos. KF201504 and KF201706), the Postdoctoral Science-research Developmental Foundation of Heilongjiang Province (grant no. LBH-Q12030) and the Natural Science Foundation of Heilongjiang Province of China (grant no. D201266).

\section{Availability of data and materials}

The datasets used and/or analyzed during the current study are available from the corresponding author on reasonable request.

\section{Authors' contributions}

HM and JY designed the research; HM, JL, SZ, SL, MZ and YJ performed the experiments; HM analyzed the data; HM and JL wrote the paper; and all authors read and approved the final manuscript.

\section{Ethics approval and consent to participate}

The present study was approved by the Ethical Committee of The Second Affiliated Hospital of Harbin Medical University (Harbin, China).

\section{Consent for publication}

Not applicable.

\section{Competing interests}

The authors declare no that they have no competing interests.

\section{References}

1. Westrate LM, Lee JE, Prinz WA and Voeltz GK: Form follows function: The importance of endoplasmic reticulum shape. Annu Rev Biochem 84: 791-811, 2015.
2. Yang L, Zhao D, Ren J and Yang J: Endoplasmic reticulum stress and protein quality control in diabetic cardiomyopathy. Biochim Biophys Acta 1852: 209-218, 2015.

3. Zhou H, Zhu J, Yue S, Lu L, Busuttil RW, Kupiec-Weglinski JW, Wang X and Zhai Y: The dichotomy of endoplasmic reticulum stress response in liver ischemia-reperfusion injury. Transplantation 100: 365-372, 2016.

4. Iurlaro R and Muñoz-Pinedo C: Cell death induced by endoplasmic reticulum stress. FEBS J 283: 2640-2652, 2016.

5. Liu J, Ren F, Cheng Q, Bai L, Shen X, Gao F, Busuttil RW, Kupiec-Weglinski JW and Zhai Y: Endoplasmic reticulum stress modulates liver inflammatory immune response in the pathogenesis of liver ischemia and reperfusion injury. Transplantation 94: 211-217, 2012.

6. Hou JY, Liu Y, Liu L and Li XM: Protective effect of hyperoside on cardiac ischemia reperfusion injury through inhibition of ER stress and activation of Nrf2 signaling. Asian Pac J Trop Med 9: 76-80, 2016.

7. Nakka VP, Gusain A and Raghubir R: Endoplasmic reticulum stress plays critical role in brain damage after cerebral ischemia/reperfusion in rats. Neurotox Res 17: 189-202, 2010

8. Kersten S: Integrated physiology and systems biology of PPAR $\alpha$. Mol Metab 3: 354-371, 2014.

9. Nan YM, Wang RQ and Fu N: Peroxisome proliferator-activated receptor $\alpha$, a potential therapeutic target for alcoholic liver disease. World J Gastroenterol 20: 8055-8060, 2014.

10. Su Q, Baker C, Christian P, Naples M, Tong X, Zhang K, Santha $\mathrm{M}$ and Adeli K: Hepatic mitochondrial and ER stress induced by defective PPAR $\alpha$ signaling in the pathogenesis of hepatic steatosis. Am J Physiol Endocrinol Metabol 306: E1264-E1273, 2014.

11. Palomer X, Capdevila-Busquets E, Garreta G, Davidson MM and Vázquez-Carrera M: PPAR $\alpha$ attenuates palmitate-induced endoplasmic reticulum stress in human cardiac cells by enhancing AMPK activity. Clin Investig Arterioscler 26: 255-267, 2014 (In Spanish).

12. Mo H, Zhao S, Luo J and Yuan J: PPAR $\alpha$ activation by fenofibrate protects against acute myocardial ischemia/reperfusion injury by inhibiting mitochondrial apoptosis. Int J Clin Exp Pathol 9: 10955-10964, 2016.

13. Yuan J, Wu J and Han ZG: Fenofibrate improves energy metabolism and attenuates isoproterenol induced acute myocardial ischemic injury in rats via PPAR alpha activation. Zhonghua Xin Xue Guan Bing Za Zhi 36: 847-850, 2008 (In Chinese).

14. Livak KJ and Schmittgen TD: Analysis of relative gene expression data using real-time quantitative PCR and the 2(-Delta Delta C(T)) method. Methods 25: 402-408, 2001.

15. Jones MG, Anderson KM, Wilson PW, Kannel WB, Wagner NB and Wagner GS: Prognostic use of a QRS scoring system after hospital discharge for initial acute myocardial infarction in the Framingham cohort. Am J Cardiol 66: 546-550, 1990.

16. Tjandrawidjaja MC, Fu Y, Westerhout CM, Wagner GS, Granger CB and Armstrong PW; APEX-AMI Investigators: Usefulness of the QRS score as a strong prognostic marker in patients discharged after undergoing primary percutaneous coronary intervention for ST-segment elevation myocardial infarction. Am J Cardiol 106: 630-634, 2010.

17. Shiomi H, Kosuge M, Morimoto T, Watanabe H, Taniguchi T, Nakatsuma K, Toyota T, Yamamoto E, Shizuta S, Tada T, et al: QRS score at presentation electrocardiogram is correlated with infarct size and mortality in ST-segment elevation myocardial infarction patients undergoing primary percutaneous coronary intervention. Circ J 81: 1129-1136, 2017.

18. Blunt BC, Creek AT, Henderson DC and Hofmann PA: $\mathrm{H} 2 \mathrm{O} 2$ activation of HSP $25 / 27$ protects desmin from calpain proteolysis in rat ventricular myocytes. Am J Physiol 293: 1518-1525, 2007.

19. Turer AT and Hill JA: Pathogenesis of myocardial ischemia-reperfusion injury and rationale for therapy. Am J Cardiol 106: 360-368, 2010.

20. Singh RB, Chohan PK, Dhalla NS and Netticadan T: The sarcoplasmic reticulum proteins are targets for calpain action in the ischemic-reperfused heart. J Mol Cell Cardiol 37: 101-110, 2004.

21. Kumarapeli AR and Wang X: Genetic modification of the heart: Chaperones and the cytoskeleton. J Mol Cell Cardiol 37: 1097-1109, 2004.

22. Chohan PK, Singh RB, Dhalla NS and Netticadan T: L-arginine administration recovers sarcoplasmic reticulum function in ischemic reperfused hearts by preventing calpain activation. Cardiovasc Res 69: 152-163, 2006. 
23. Zhang CM, Gao L, Zheng YJ and Yang HT: Berbamine protects the heart from ischemia/reperfusion injury by maintaining cytosolic $\mathrm{Ca}(2+)$ homeostasis and preventing calpain activation. Circ J 76: 1993-2002, 2012.

24. French JP, Quindry JC, Falk DJ, Staib JL, Lee Y, Wang KKW and Powers SK: Ischemia-reperfusion-induced calpain activation and SERCA2a degradation are attenuated by exercise training and calpain inhibition. Am J Physiol Heart Circulat Physiol 290 : 128-136, 2006

25. Wilding JR, Joubert F, de Araujo C, Fortin D, Novotova M, Veksler V and Ventura-Clapier R: Altered energy transfer from mitochondria to sarcoplasmic reticulum after cytoarchitectural perturbations in mice hearts. J Physiol 575: 191-200, 2006.

26. Calaghan SC, Guennec JYL and White E: Cytoskeletal modulation of electrical and mechanical activity in cardiac myocytes Prog Biophys Mol Biol 84: 29-59, 2004.

27. Krebs J, Agellon LB and Michalak $\mathrm{M}^{\mathrm{Ca}^{2+}}$ homeostasis and endoplasmic reticulum (ER) stress: An integrated view of calcium signaling. Biochem Biophys Res Communicat 460: 114-121, 2015.

28. Kim J, Choi TG, Ding Y, Kim Y, Ha KS, Lee KH, Kang I, Ha J, Kaufman RJ,Lee J, et al: Overexpressed cyclophilin B suppresses apoptosis associated with ROS and $\mathrm{Ca} 2+$ homeostasis after ER stress. J Cell Sci 121: 3636-3648, 2008.

29. Li Y, Zhu W, Tao J, Xin P, Liu M, Li J and Wei M: Fasudil protects the heart against ischemia-reperfusion injury by attenuating endoplasmic reticulum stress and modulating SERCA activity: The differential role for PI3K/Akt and JAK2/STAT3 signaling pathways. PLoS One 7: e48115, 2012.

30. Armstrong SC, Shivell CL and Ganote CE: Sarcolemmal blebs and osmotic fragility as correlates of irreversible ischemic injury in preconditioned isolated rabbit cardiomyocytes 弥. $\mathrm{J}$ Mol Cell Cardiol 33: 149-160, 2001.
31. Pavli M, Farmaki E, Merkourea S, Vastardis H, Sklavounou A, Tzerbos F and Chatzistamou I: Endoplasmic reticulum stress-associated chaperones, Bip/GRP78 and calnexin are overexpressed in keratocystic odontogenic tumours. J Oral Maxillofac Res 5: e3, 2014.

32. Minamino T, Komuro I and Kitakaze M: Endoplasmic reticulum stress as a therapeutic target in cardiovascular disease. Circ Res 107: 1071-1082, 2010.

33. Lam VH, Zhang L, Huqi A, Fukushima A, Tanner BA, Onay-Besikci A, Keung W, Kantor PF, Jaswal JS, Rebeyka IM and Lopaschuk GD: Activating PPAR $\alpha$ prevents post-ischemic contractile dysfunction in hypertrophied neonatal hearts. Circ Res 117: 41-51, 2015.

34. Pantazi E, Folch-Puy E, Bejaoui M, Panisello A, Varela AT, Rolo AP, Palmeira CM and Roselló-Catafau J: PPAR agonist WY-14643 induces SIRT1 activity in rat fatty liver ischemia-reperfusion injury. Biomed Res Int 2015: 894679, 2015.

35. Luciani DS, Gwiazda KS, Yang TL, Kalynyak TB, Bychkivska Y, Frey MH, Jeffrey KD, Sampaio AV, Underhill TM and Johnson JD: Roles of IP3R and RyR Ca2+ channels in endoplasmic reticulum stress and beta-cell death. Diabetes 58: 422-432, 2009. Attribution-NonCommercial-NoDerivatives 4.0 International (CC BY-NC-ND 4.0) License. 